\title{
MOAD: Modeling Observation-based Approximate Dependency
}

\author{
Seongmin Lee \\ KAIST \\ Daejeon, Republic of Korea \\ bohrok@kaist.ac.kr
}

\author{
David Binkley \\ Loyola University Maryland \\ Baltimore, United States \\ binkley@cs.loyola.edu
}

\author{
Robert Feldt \\ Chalmers University of Technology \\ Gothenburg, Sweden \\ robert.feldt@chalmers.se
}

\author{
Nicolas Gold \\ University College London \\ London, United Kingdom \\ n.gold@ucl.ac.uk
}

\author{
Shin Yoo \\ KAIST \\ Daejeon, Republic of Korea \\ shin.yoo@kaist.ac.kr
}

\begin{abstract}
While dependency analysis is foundational to many applications of program analysis, the static nature of many existing techniques presents challenges such as limited scalability and inability to cope with multi-lingual systems. We present a novel dependency analysis technique that aims to approximate program dependency from a relatively small number of perturbed executions. Our technique, called MOAD (Modeling Observation-based Approximate Dependency), reformulates program dependency as the likelihood that one program element is dependent on another, instead of a more classical Boolean relationship. MOAD generates a set of program variants by deleting parts of the source code, and executes them while observing the impacts of the deletions on various program points. From these observations, MOAD infers a model of program dependency that captures the dependency relationship between the modification and observation points. While MOAD is a purely dynamic dependency analysis technique similar to Observation Based Slicing (ORBS), it does not require iterative deletions. Rather, MOAD makes a much smaller number of multiple, independent observations in parallel and infers dependency relationships for multiple program elements simultaneously, significantly reducing the cost of dynamic dependency analysis. We evaluate MOAD by instantiating program slices from the obtained probabilistic dependency model. Compared to ORBS, MOAD's model construction requires only $18.7 \%$ of the observations used by ORBS, while its slices are only $16 \%$ larger than the corresponding ORBS slice, on average.
\end{abstract}

\section{INTRODUCTION}

Understanding dependencies between program elements is a fundamental task in software engineering [1], [2]. It provides a basis for many software engineering tasks including program comprehension [3], software testing [4], maintenance [5], [6], refactoring [7], security [8], and debugging [9]. The traditional static approach based on dependence graphs [10] has been widely adopted but suffers from issues such as its inability to handle multi-lingual systems (combining analyses for multiple languages is too complicated) and limited scalability (partial analysis of a large system is not viable using static approaches that require whole-program analyses).

Observation Based Slicing (ORBS) [11]-[14] was designed to overcome these issues. ORBS applies speculative deletions iteratively to the program under analysis, and observes whether the latest applied deletion is viable (i.e., the code compiles after deletion) and is unrelated to the slicing criteria (i.e., the variable of interest shows the same behaviour after deletion with respect to a test suite). When deletions are made at the line of text level, ORBS can be entirely language agnostic [11] and can analyse files for which the grammar is unavailable/unknown and languages with unconventional semantics, such as Picture Description Languages (PDLs) [15]. Despite its benefits, ORBS has one clear drawback: the cost of analysis. Being a purely dynamic approach, it iteratively attempts to validate its speculative deletion of program elements via compilations and test executions. As such, it can incur a significant cost.

This paper investigates whether it is possible to perform approximate dependency analysis dynamically at a lower cost. A precise dependency analysis can report whether program element A depends on program element B or not: the outcome is Boolean. An approximate dependency analysis instead reports the likelihood that A depends on B: the outcome is a real number. While probabilistic program dependency analysis techniques have been proposed before [2] they require an initial static analysis which is then extended with probabilistic information based on test executions. We conjecture that a more general, approximate dependency analysis based on dynamic observations can still be useful in many program analysis contexts while being significantly less costly.

The approximate nature of our approach stems from the fact that it infers a stochastic model of program dependencies. Unlike ORBS which performs iterative deletions to analyse program dependency with respect to a single program element (i.e., the slicing criterion), our technique, MOAD (Modeling Observation-based Approximate Dependency), learns an approximate model of program dependence from (a smaller number of) dynamic observations. Intuitively, ORBS makes a single slice increasingly more accurate by iterative deletion. MOAD, however, employs a set of deletions that can be treated independently. For each, it observes multiple program elements. This approach introduces the following benefits:

- MOAD requires many fewer observations than ORBS, as 
it infers the relationships between individual deletions and thus the dependency, instead of uncovering dependence information by iteratively deleting until it arrives at a one-minimal slice [11].

- The output of MOAD can be used to construct multiple slices, whereas a single ORBS run produces a single slice.

- Moreover, since the observations required by MOAD are independent from each other, MOAD is inherently parallel.

To evaluate our claims, we have implemented MOAD and performed dependency analysis against a benchmark suite of programs that have been widely used in the slicing literature. We evaluate the viability and the accuracy of MOAD by instantiating concrete slices based on the outcome of MOAD: program element $\mathrm{A}$ is in the backward slice of program element B iff the reported likelihood of B depending on A is greater than a threshold value. A comparison to a baseline random slicing technique shows that MOAD is indeed learning program dependencies; a comparison to ORBS slices shows that MOAD can produce slices that are only $16 \%$ larger than ORBS slices, while using only $18.7 \%$ of the observations. We also investigate various ways to construct the observation sets, as well as the impact of different inference models. Note that dependency modeling is more general than program slicing (and has other uses [2]). However, we leave these additional use cases for future work and focus here on slicing as a representative approach.

The technical contributions of this paper are as follows:

- We introduce the concept of learning approximate dependency analysis, which transforms the dependency relationship from Boolean to probabilistic.

- We present MOAD, a technique that models approximate program dependency, and describe the essential components: how to generate observations, and how to infer models of program dependency.

- We conduct an empirical evaluation of MOAD via program slices instantiated from the learned models.

The rest of this paper is organised as follows. Section II introduces the concept of approximate dependency analysis, and explains how it relates to the existing slicing technique ORBS. Section III introduces MOAD, a technique that aims to model approximate dependency, and how we can use it for slicing by instantiating program slices from the learned dependency models. Section IV presents the set-up of empirical evaluation, the results of which are reported in Section V. Section VI contains discussions of our findings and potential future work. Section VII presents the related work, and Section VIII concludes.

\section{Approximating Program Dependency}

Program dependencies are dependence relations that hold between elements of a program (e.g., statements, expressions, or variables). If the computation of one element directly or indirectly affects the computation of another element we consider them to be dependent. A plethora of techniques have been proposed to capture and model dependence information.
Often these techniques are static and require parsing and detailed analysis of program elements based on the semantics of the programming language in question. The outcome of the analysis typically captures a binary dependence relation where two program elements either have or may not have a dependence relation. While dynamic dependence analysis approaches have been proposed, they typically annotate an already extracted static model with probabilities based on concrete executions [2] (static-then-annotate) or do not build any explicit model at all [11].

One downside of the static and static-then-annotate approaches is that they cannot handle heterogeneous systems, some of whose components are either binary, or written in multiple languages and file formats. Even if it is possible in theory to combine analyses of multiple languages and formats, concrete tools actually support only a fixed and typically small selection. An additional practical problem is that they need to duplicate the early stages of multiple compiler tool chains.

Observation-based slicing [11] (ORBS) addresses these problems and allows language-agnostic dynamic slicing without detailed semantic knowledge, by reusing the build chain. An implementation of ORBS is also trivial and requires very few lines of code. However, ORBS does not learn a general model of the program components and their relations. Given a target slicing criterion, it simply creates a slice through iterative build and execute cycles. The process has to be repeated if another slicing criterion is selected for analysis, which typically happens in fault diagnosis or debugging applications.

This paper proposes a middle ground that leverages the minimal requirements, general applicability, and easy implementation of lightweight dynamic analysis techniques such as ORBS, while modeling dependence relations approximately. By not requiring any detailed syntactic or semantic knowledge of the components or programming languages involved, we can support heterogeneous systems with a very general approach and tool. Since our model building is based on a few, specific, dynamic executions it can only approximate the complete dependency information. However, explicitly building such probabilistic models brings potential advantage over model-free, dynamic approaches such as ORBS, which do not learn anything between invocations. We posit that there exists an interesting and possibly complementary tradeoff between what we propose and existing program analysis methods.

While the idea of approximate program dependency modeling is a general one, we focus below on a simple instantiation of it that targets slicing. This allows us to study the potential benefits compared to a well-known and general technique. Thus in the following we thus describe MOAD in the context of program slicing.

\section{MOAD: MODELING OBSERVATION-BASED APPROXIMATE DEPENDENCY}

This section first overviews the key terminology used to describe MOAD, before presenting its two phases: the observation phase and the inference phase. The output of the first 
phase is a set of observations. These observations form the input to the inference phase, which builds an inference model $M$ that aims to capture the dependence within the program. As a case study, we illustrate $M$ by inferring program slices [16], [17]. In the next section we compare the inferred slices with those produced using ORBS [11], [12].

\section{A. Terminology}

Our approach is dynamic in nature and thus, in addition to a program, $P$, it takes a set of test inputs, $I$. We identify within $P$ a set of deletable units $U=\left\{u_{1}, \ldots, u_{|U|}\right\}$. For example, $U$ might be composed of lines of text, program statements, or blocks of code within a program. We subsequently create sub-programs of $P, P^{\prime}$, by deleting one or more units from $P$. To support the inference process, we represent a sub-program as a boolean vector, called deletion, which has one entry for each unit. In this vector deleted units are assigned the value TRUE and retained units are assigned the value FALSE.

Borrowing the notion from program slicing [16], [17], we assess the impact of deleting various units at a set of (slicing) criteria, $C=\left\{c_{1}, \ldots, c_{|C|}\right\}$. Each criteria $c_{i}$ includes a program location (e.g., a line number) and a variable of interest (e.g., the variable updated by an assignment statement). To determine the impact of deleting a given unit, we observe the sequence of values produced by each criterion. The result is a boolean vector, called response, that has one entry per criterion. The entry $c_{i}$ has the value TRUE iff the sequence of values produced for $c_{i}$ is unaffected by the deletion (with respect to the sequence produced by the original program). The key assumption here is that, if the deletion of unit $u_{a}$ brings about a change in the the trajectory for criterion $c_{b}$, then the criterion $c_{b}$ likely depends on (some part of) unit $u_{a}$.

\section{B. Observation Phase}

The core of the first phase is a deletion generation scheme, which generates the set of deletions used to produce program mutants. Our experiments consider the two $n$-hot deletions schemes: 1-hot and 2-hot. The first, 1-hot generates $|U|$ deletions where each deletion removes exactly one unit. In other words, it generates all of the one-hot encoding vectors of length $|U|$. The second, 2-hot subsumes 1-hot; it considers both all single units and all pairs of units. (Because 2-hot involves dramatically more deletions than 1-hot, in Section V-D we also consider the impact of sampling the 2-hot data.)

Algorithm 1 describes the observation phase. Its input includes, $P$, the program under study, $I$, the input test suite, and a deletion generation scheme, GENSCHEME. The algorithm assumes that $P$ has been annotated to output a sequence of values capturing each slicing criterion, similar to ORBS [11]. The algorithm first initializes the output observation set, $O$, to the empty set, $E$ to the expected output sequence for each criteria (collected from the execution of unmodified $P$ and $I$ ), and the set of deletions generated using the given scheme. Subsequently, Lines 4-9 process each deletion by first using the function APPLY to generate sub-program $P^{\prime}$ composed of only the non-deleted units (omitting those those units whose

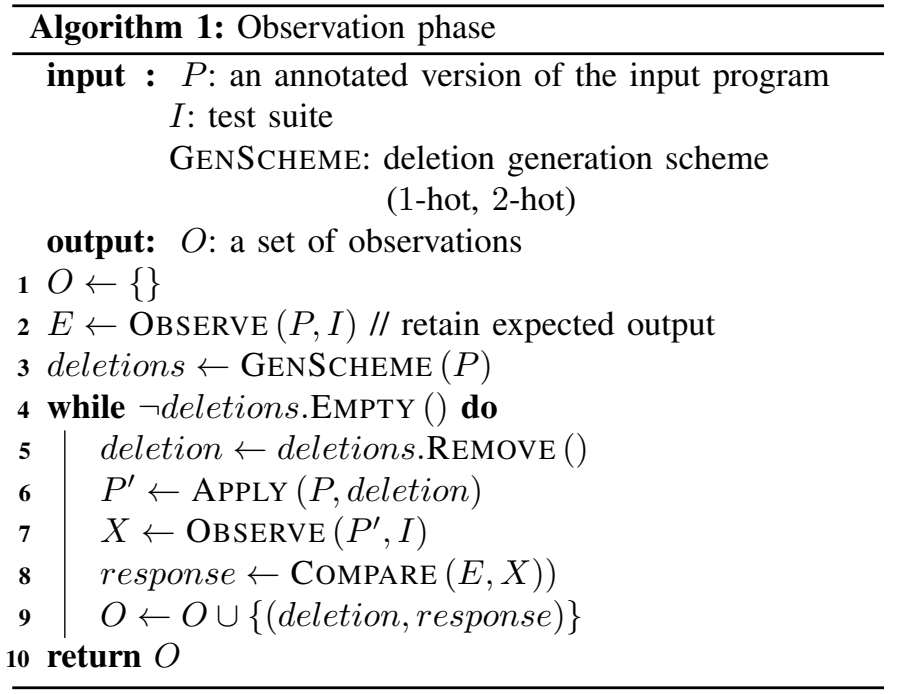

value in deletion is TRUE). Next, function OBSERVE executes $P^{\prime}$ using set of inputs $I$ to produce the trajectories for each criterion as produced by the annotations in $P$. The final step compares the expected output $E$ with the output of $P^{\prime}$, to produce the result vector response. Note that $P^{\prime}$ may fail to compile in which case its outputs will fail to match the expected output. The response is paired with the deletion and recorded in the set of observations to be returned by the algorithm. The main difference between Algorithm 1 and ORBS is that while ORBS is a cumulative process to produce a slice for one slicing criterion, each observation in Algorithm 1 is independent, thus it can be done in parallel.

To illustrate Algorithm 1, consider a program $P$ with three statements $S_{1}, S_{2}$, and $S_{3}$ using the 1-hot generation scheme. With three statements, 1 -hot produces the three deletions $\{$ True, False, False $\},\{$ False, True, False $\}$, and $\{$ False, False, True $\}$. Applying the first deletion to $P$ produces the two statement program $S_{2} ; S_{3}$, which is then observed (compiled and executed using input $I$ ).

\section{Inference phase}

The inference phase, described in Algorithm 2, infers the slices for each criterion. The algorithm uses the set of observations, $O$, (output by the first phase) to train an inference model, $M: C \rightarrow D=$ Boolean $^{|U|}$, where $D$ is the set of possible deletions. Then, for each slicing criterion, $M$ is used to infer a set of deletions, which is applied to the original program to produce the slice.

The key assumption made in the inference process is that if deleting the unit $u_{m}$ changes the trajectory of the slicing criterion $c_{k}$, then $u_{m}$ is likely to be in the slice of $c_{k}$. While this connection is straightforward, this data tends to overestimate deletability for the 1-hot data. For example when either of two statements can be deleted, but not both [11]. In contrast, for $n$-hot when $n$ is greater than one, it is possible that not all of the deleted units influence $c_{k}$.

The remainder of this subsection details three different inference models studied in the next section. As a notational 




convenience, hereafter we use " 0 " and " 1 " to denote "FALSE" and TRUE", respectively.

Once Success (O): The Once Success model explicitly follows the aforementioned assumption. Assume subprogram $P^{\prime}$ is obtained from program $P$ by removing deletion unit $u_{m}$. If $P^{\prime}$ preserves the trajectory of the slicing criterion $c_{k}$, the model removes $u_{m}$ from the slice of $c_{k}$. More formally, the Once Success model, $M_{\mathbb{O}}$, trained with observations $O$, infers the deletion of the slice of $c_{k}$ as follows:

$M_{\mathbb{O}}\left(c_{k}\right)[m]=\left\{\begin{array}{l}1, \text { if } \exists(d, r) \in O \text { s.t. } d[m]=1 \text { and } r[k]=1 \\ 0, \text { otherwise }\end{array}\right.$

where $d[m]$ represents the $m^{t h}$ element of deletion vector $s$ and $r[k]$ represents the $k^{t h}$ element of response vector $r$. Thus, $d[m]=1$ and $r[k]=1$ represents that unit $m$ has been deleted and the response for criterion $k$ is unchanged.

Logistic $(\mathbb{L})$ : The Logistic model regards the response element, $r[k]$ (for slicing criterion $c_{k}$ ) as a dependent variable and the elements of the deletion, $d$, as the independent variables. Because the variables are binary values, they are modeled using logistic regression,

$$
r[k] \approx L\left(d, \beta_{k}\right)
$$

where the elements of $\beta_{k}$ are the coefficients of the regression. The sign of each coefficient is used to determine if the corresponding unit is removed to preserve the slicing criterion $c_{k}$. If $\beta_{k}[m]$, the $m^{t h}$ coefficient of $\beta_{k}$, has a positive value, $u_{m}$ is more likely to be removed from the slice, while a negative value indicates that $u_{m}$ is less likely to be removed from the slice. More formally, $M_{\mathbb{L}}$, the Logistic model, infers the deletion vector for the slice taken with respect to $c_{k}$ as follows:

$$
M_{\mathbb{L}}\left(c_{k}\right)[m]=\left\{\begin{array}{l}
0, \text { if } \beta_{k}[m] \leq 0 \\
1, \text { if } \beta_{k}[m]>0
\end{array} .\right.
$$

Bayesian $(\mathbb{B})$ : The final model we consider uses Bayesian inference. We assume that $P\left(c_{k} \mid u_{m}\right)$ denotes the conditional probability of preserving the trajectory of $c_{k}$ when the unit $u_{m}$ has been deleted. From the observations, $O$, we estimate $\hat{P}\left(c_{k} \mid u_{m}\right)$ as follows:

$$
\begin{aligned}
P\left(c_{k} \mid u_{m}\right) & =P\left(\text { preserves trajectory of } c_{k} \mid u_{m} \text { has been deleted }\right) \\
& =P(r[k]=1 \mid d[m]=1) \\
& =\frac{P(r[k]=1, d[m]=1)}{P(d[m]=1)} \\
\hat{P}\left(c_{k} \mid u_{m}\right) & =\frac{\#(r[k]=1 \text { and } d[m]=1) /|O|}{\#(d[m]=1) /|O|} \\
& =\frac{\#(r[k]=1 \text { and } d[m]=1)}{\#(d[m]=1)},
\end{aligned}
$$

where \#(cond) is a number of observations in $O$ satisfying the condition cond. Formally, $M_{\mathbb{B}}$, the Bayesian model, infers the deletion of the slice of $c_{k}$ as follows:

$M_{\mathbb{B}}\left(c_{k}\right)[m]=\left\{\begin{array}{l}0, \text { if } \hat{P}\left(c_{k} \mid u_{m}\right) \leq \mu_{i \in\{1 . .|U|\}}\left(\hat{P}\left(c_{k} \mid u_{i}\right)\right) \\ 1, \text { if } \hat{P}\left(c_{k} \mid u_{m}\right)>\mu_{i \in\{1 . .|U|\}}\left(\hat{P}\left(c_{k} \mid u_{i}\right)\right),\end{array}\right.$

where $\mu_{i \in\{1 . .|U|\}}\left(\hat{P}\left(c_{k} \mid u_{i}\right)\right)$ is an average value of the estimated probability.

\section{EXPERIMENT SETUP}

\section{A. Research Questions}

We evaluate MOAD by investigating the following four research questions.

\section{RQ1. Viability: Do the learned models capture program dependence information?}

To ascertain if our approach is viable we compare the learned models' ability to produce slices against that of a random slicer. If none of the models can outperform a random slicer then there is no reason to consider them further.

RQ2. Impact of the inference model: Assuming that more than one model is viable, how does the performance of the viable models compare?

To study RQ2 we consider the ability of each model to compute program slices. Because the models are trained with runtime information, they approximate dynamic slices. The most closely related dynamic slicing approach is observational slicing. As benchmarks we consider the slices produced by two observational slicing implementations W-ORBS [11] and T-ORBS [13], [14]. While these two are expected to produce more accurate slices than MOAD, they are also expected to take longer to do so.

When considering RQ2, performance is compared in terms of both slice precision, measured in lines of code, and slicing effort, measured as the number of observations required.

RQ3. Performance compared to ORBS: For the best inference model, how well does MOAD perform compared to ORBS?

Based on the results from RQ2, we compare the performance of ORBS and MOAD when using the best of the viable models. Parallel to RQ2, effort is measured in terms of the number of observations required. However, for precision, we take a more refined approach and count both missing and excess lines relative to the W-ORBS slice. 
RQ4. Sampling effect: How feasible is using only a sample of the 2-hot data?

The primary goal of RQ4 is to determine if there is a sweet spot in the analysis that best balances precision and effort. Our expectation here is that a model trained with more observations will have higher precision, but require greater effort. To investigate RQ4, we consider ten different sample sizes when sampling from each program's set of 2-hot observations. The sizes include $10 \%, 20 \%, \ldots, 100 \%$ of the 2 -hot observations. Since the performance might be biased by a particular sample, we repeat the sampling for each size ten times.

\section{B. Subjects}

\begin{tabular}{l|rrr}
\hline Subject & SLoC & $|U|$ & $|C|$ \\
\hline mbe & 64 & 45 & 16 \\
mug & 61 & 44 & 13 \\
wc & 46 & 33 & 17 \\
\hline prttok & 410 & 388 & 98 \\
prttok2 & 387 & 364 & 75 \\
replace & 508 & 465 & 253 \\
sched & 283 & 252 & 75 \\
sched2 & 276 & 248 & 81 \\
totinfo & 314 & 227 & 210 \\
tcas & 152 & 110 & 62 \\
\hline
\end{tabular}

TABLE I: The statistics of experiment subjects' properties.

Table I shows the programs we study. It includes the number of non-comment-non-blank lines (SLoC), the number of units, and the number of criteria used. The first three subjects, mbe, mug, wc, are small, well known, programs that have well studied semantics. This makes them amenable to careful precise study. Furthermore, the first two raise specific challenges to dependence analysis and thus serve to highlight the pros and cons of our approximation technique. In addition, we study the Siemens suite [18], to see how our technique works on ordinary C code. The Siemens suite is used in lieu of larger programs because it is possible to exhaustively compute all the slices of each program (for all scalar slicing criteria). This removes any slice selection bias from the data.

\section{Observation-based Slicing (ORBS)}

We use Observation-Based Slicing (ORBS) [11] as a benchmark approach to evaluate the performance of MOAD. ORBS is a dynamic program slicing technique based on direct observation of program semantics (when executing the program on a chosen test suite). An ORBS slicer performs iterative, speculative deletion of parts of the code. Each deletion is made permanent if it preserves the trajectory of values computed at the slicing criterion.

The original ORBS implementation [11], slices source code at the line-of-text level. We refer to this algorithm as W-ORBS where the ' $\mathrm{W}$ ' captures the use of a deletion window, in which W-ORBS considers the deletion of a sequence of consecutive lines of text. In addition to a performance advantages, the use of a deletion window enables W-ORBS to delete lines that can only be deleted together (e.g., the pair of brackets that enclose an empty block). Applied to line $l_{i}$, W-ORBS attempts to delete from one to $k$ lines (i.e., from $\left\{l_{i}\right\}$ to $\left\{l_{i}, \ldots, l_{i+k-1}\right\}$ ). If it successfully deletes $j$ lines (i.e., $\left\{l_{i}, \ldots, l_{i+j-1}\right\}$ ), the deletion continues with line $l_{i+j}$; if all $k$ attempts fail, the deletion continues with line $l_{i+1}$. Thus after each successful deletion, W-ORBS moves onto the next target source code line (skipping over the deleted lines), while after each unsuccessful deletion it reverts the deletion before moving on to the next line of the file. W-ORBS performs multiple passes over the code until it cannot delete anything further, producing a 1minimal line slice [11].

A recent variation of W-ORBS, T-ORBS [13], [14] works with a tree-based representation. T-ORBS performs a breadthfirst tree traversal using a work list. For each node, $n$, it attempts to delete the subtree rooted at $n$. If the resulting program produces the same trajectory for the slicing criterion then the subtree is permanently deleted. Otherwise, its children are appended on the work list for later consideration. The T-ORBS implementation we used employs SrcML [19] to produce an XML tree from a program. It is important to note that we modified the original T-ORBS algorithm to attempt to delete only those syntactic elements that are considered by MOAD; in this case statements. Our motivation for modifying T-ORBS to attempt the deletion of only those syntactic elements that correspond to the units considered by MOAD is to provide an apples-to-apples comparison. Otherwise T-ORBS takes considerably longer as it has to consider numerous subtrees that represent only a subset of the statement. Doing so requires introducing a small amount of language information into an otherwise language agnostic algorithm but provides a better basis for comparison (and as a consequence also brings a dramatic speedup).

\section{Configuration}

In the initial experiments, the units considered by MOAD are programming language statements. We use SrcML (version 0.9.5) [19], an XML-based multi-language parsing tool, to identify statements. SrcML enables our approach to be applied to any programming language, including multi-lingual programs, that SrcML can process. Other than this dependence, our algorithm is language independent.

The set of slicing criteria considered consist of all scalar (char, int, float, etc.) assignments. We use Clang (version 3.8) [20] to insert logging statements for each slicing criterion. These statements are responsible for outputting the sequence of values computed for the criteria. A slicer's goal is to preserve this sequence while removing unnecessary code.

As a benchmark, we apply both W-ORBS (with maximum window size of three) and (the modified) T-ORBS to our subjects.

The experiments were performed under Ubuntu 16.04, on an Intel(R) Core(TM) i7 CPU with 32GB of memory using gec version 5.5.0.

\section{E. Metrics}

There are various metrics used to measure and to compare the performance of the slicing techniques. 
- $W S_{k}, T S_{k}$, and $M S_{k}$ : The slice taken with respect to criterion $c_{k}$ as computed by W-ORBS, T-ORBS, and MOAD, respectively.

- miss: Given a reference slice (e.g., $W S_{k}$ ) and an inferred slice (e.g., $M S_{k}$ ) the number of units that should have been removed (i.e., that were missed) from the inferred slice. In other words, it is the number of units in the inferred slice that are not in the reference slice.

- excess: Given a reference slice (e.g., $W S_{k}$ ) and an inferred slice (e.g., $M S_{k}$ ) the number of units that are excessively removed from the inferred slice. In other words, it is the number of units in the reference slice that are not in the inferred slice.

By design T-ORBS and MOAD share the same set of deletable units. Thus, we can calculate miss and excess directly applying a set difference between the set of units making up the slice. Since W-ORBS modifies the source code at the line-of-text level, the same method is not viable when comparing W-ORBS and MOAD. Instead, we use a python difflib module to calculate miss and excess at the line level.

\section{RESULTS}

\section{A. Viability}

To answer RQ1, we first create a random slicer. Our implementation randomly deletes each unit with a probability of 0.5 . For every slicing criterion in every subject program, we run the random slicer ten times and check whether the slice generated preserves the trajectory of the slicing criterion. With 900 slicing criteria (see Table I) spread across the ten subject programs, the random slicer generates 9,000 slices in total. Only fifteen of the random slices compile, and none of them preserve the trajectory of the given slicing criterion. This result clearly indicates that it is very unlikely to produce a slice by chance.

\begin{tabular}{|c|c|c|c|c|}
\hline \multirow{2}{*}{ Subject } & \multirow{2}{*}{$\begin{array}{c}\text { Deletion } \\
\text { Gen. Scheme }\end{array}$} & \multicolumn{3}{|c|}{ Success Rate } \\
\hline & & (1) & $\mathbb{L}$ & $\mathbb{B}$ \\
\hline \multirow{2}{*}{ mbe } & 1-hot & $100 \%$ & $100 \%$ & $100 \%$ \\
\hline & 2-hot & $100 \%$ & $100 \%$ & $100 \%$ \\
\hline \multirow{2}{*}{ mug } & 1-hot & $100 \%$ & $100 \%$ & $100 \%$ \\
\hline & 2-hot & $100 \%$ & $100 \%$ & $100 \%$ \\
\hline \multirow{2}{*}{ wc } & 1-hot & $100 \%$ & $100 \%$ & $100 \%$ \\
\hline & 2-hot & $88 \%$ & $76 \%$ & $100 \%$ \\
\hline \multirow{2}{*}{ prttok } & 1-hot & $03 \%$ & $04 \%$ & $11 \%$ \\
\hline & 2-hot & $03 \%$ & $03 \%$ & $11 \%$ \\
\hline \multirow{2}{*}{ prttok2 } & 1-hot & $72 \%$ & $19 \%$ & $77 \%$ \\
\hline & 2-hot & $63 \%$ & $13 \%$ & $67 \%$ \\
\hline \multirow{2}{*}{ replace } & 1-hot & $7 \%$ & $31 \%$ & $28 \%$ \\
\hline & 2-hot & $3 \%$ & $13 \%$ & $31 \%$ \\
\hline \multirow{2}{*}{ sched } & 1-hot & $48 \%$ & $47 \%$ & $41 \%$ \\
\hline & 2-hot & $39 \%$ & $35 \%$ & $43 \%$ \\
\hline \multirow{2}{*}{ sched2 } & 1-hot & $30 \%$ & $26 \%$ & $28 \%$ \\
\hline & 2-hot & $17 \%$ & $26 \%$ & $28 \%$ \\
\hline \multirow{2}{*}{ totinfo } & 1-hot & $52 \%$ & $50 \%$ & $62 \%$ \\
\hline & 2-hot & $32 \%$ & $10 \%$ & $65 \%$ \\
\hline \multirow[t]{2}{*}{ tcas } & 1-hot & $48 \%$ & $90 \%$ & $48 \%$ \\
\hline & 2-hot & $26 \%$ & $68 \%$ & $48 \%$ \\
\hline
\end{tabular}

TABLE II: MOAD's success rate on the ten test subjects

In contrast, Table II shows the ability of MOAD to produce viable slices that not only compile, but also capture the desired semantics. In the table, the second column shows the deletion generation scheme used to generate the observations. Then in the remaining columns we report the success rate for each of the three inference models, $\mathbb{O}, \mathbb{L}$, and $\mathbb{B}$, as the percentage of 'slices' that preserve the desired trajectory. For the smaller programs mbe, mug, and wc, most slices preserve the trajectory successfully. For the Siemens suite $42 \%$ of the generated slices preserve the trajectory.

In the table, prttok shows a particularly low success rate. Investigating this, we found that the root cause was two lines of code, shown in the snippet below, where there is a data dependence from Line 188 to Line 189. What is unusual about these two lines is that for many slices that do not depend on the value of token_ptr it is possible to individually delete either Line 188 or 189 without affecting the trajectory, but not both. Thus the model learns to remove each line. Consequently, when MOAD infers a slice it tends to unwantedly omit both lines. The result is that most trajectories change. This suggests the use of stronger statistical models (e.g., Rasmussen's Gaussian processes [21]), that can capture higher-level interaction effects between program elements.

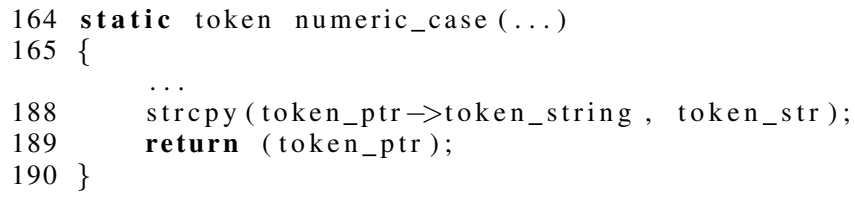

Based on these results, we answer RQ1 as follows:

RQ1. Viability: Inference models trained using dynamic observations can successfully learn program dependence.

\section{B. Model Impact}

Our initial look at the impact that a given model has focuses on the model's ability to remove units. Table III shows the average slice size, $\mu\left(W S_{k}\right), \mu\left(T S_{k}\right)$, and $\mu\left(M S_{k}\right)$, over all slicing criteria for W-ORBS, T-ORBS, and the three models used with MOAD. To facilitate inter-program comparison, the average slice sizes are given as a percentage of the original program's size. The table also shows the number of observations involved. For W-ORBS and T-ORBS this count reflects the number of compilations and executions made while computing each slice, while for MOAD the number is the number of compilations and executions used in constructing the training data.

To gain some intuition for the relative slice sizes, we first compare MOAD's average slice size with that of W-ORBS and then T-ORBS before focusing in on the impact of the individual models. Due to the approximate nature of the inference, MOAD is expected to generate larger slices than W-ORBS or T-ORBS. To normalize the data across programs, we first consider the ratio of the average slice size generated using one of the six MOAD variants (2 deletion generation scheme $\times 3$ inference model) to the average W-ORBS slice size.

Compared to W-ORBS, the results find that MOAD produces slices that are on average $45 \%$ larger than those pro- 


\begin{tabular}{|c|c|c|c|c|c|c|c|c|c|c|c|c|c|c|c|c|}
\hline \multirow[b]{2}{*}{ Subject } & \multicolumn{2}{|c|}{ W-ORBS } & \multicolumn{2}{|c|}{ T-ORBS } & \multirow[b]{2}{*}{$\left|O_{\mathrm{M}}\right|$} & \multicolumn{5}{|c|}{ 1-hot } & \multicolumn{6}{|c|}{ 2-hot } \\
\hline & $\left|O_{\mathrm{W}}\right|$ & $\mu\left(W S_{k}\right)$ & $\left|O_{\mathrm{T}}\right|$ & $\mu\left(T S_{k}\right)$ & & $\frac{\left|O_{\mathrm{M}}\right|}{\left|O_{\mathrm{W}}\right|}$ & $\frac{\left|O_{\mathrm{M}}\right|}{\left|O_{\mathrm{T}}\right|}$ & $\mathbb{O}$ & $\begin{array}{c}\mu\left(M S_{k}\right) \\
\mathbb{L}\end{array}$ & $\mathbb{B}$ & $\left|O_{\mathrm{M}}\right|$ & $\frac{\left|O_{\mathrm{M}}\right|}{\left|O_{\mathrm{W}}\right|}$ & $\frac{\left|O_{\mathrm{M}}\right|}{\left|O_{\mathrm{T}}\right|}$ & $\mathbb{O}$ & $\mu \underset{\mathbb{L}}{\mu\left(M S_{k}\right)}$ & $\mathbb{B}$ \\
\hline mbe & 6,546 & $35.1 \%$ & 1,163 & $40.6 \%$ & 47 & $0.72 \%$ & $4.04 \%$ & $46.0 \%$ & $48.2 \%$ & $45.8 \%$ & 948 & $14.5 \%$ & $81.5 \%$ & $43.4 \%$ & $48.4 \%$ & $46.2 \%$ \\
\hline mug & 4,420 & $25.6 \%$ & 969 & $33.7 \%$ & 46 & $1.04 \%$ & $4.75 \%$ & $41.7 \%$ & $51.0 \%$ & $43.1 \%$ & 904 & $20.5 \%$ & $93.3 \%$ & $35.8 \%$ & $48.3 \%$ & $45.5 \%$ \\
\hline wc & 3,843 & $23.7 \%$ & 860 & $30.3 \%$ & 35 & $0.91 \%$ & $4.07 \%$ & $37.3 \%$ & $54.6 \%$ & $46.9 \%$ & 454 & $11.8 \%$ & $52.8 \%$ & $31.6 \%$ & $44.9 \%$ & $41.7 \%$ \\
\hline prttok & 262,374 & $43.3 \%$ & 68,372 & $43.2 \%$ & 390 & $0.15 \%$ & $0.57 \%$ & $51.8 \%$ & $58.3 \%$ & $59.9 \%$ & 74,175 & $28.3 \%$ & $108.5 \%$ & $43.6 \%$ & $43.1 \%$ & $58.6 \%$ \\
\hline prttok2 & 246,668 & $37.3 \%$ & 41,324 & $38.8 \%$ & 366 & $0.15 \%$ & $0.89 \%$ & $42.6 \%$ & $66.6 \%$ & $49.6 \%$ & 65,393 & $26.5 \%$ & $158.2 \%$ & $37.6 \%$ & $56.2 \%$ & $48.8 \%$ \\
\hline replace & $1,214,230$ & $45.6 \%$ & 311,323 & $45.9 \%$ & 467 & $0.04 \%$ & $0.15 \%$ & $54.9 \%$ & $68.3 \%$ & $60.9 \%$ & 106,412 & $8.8 \%$ & $34.2 \%$ & $44.1 \%$ & $50.9 \%$ & $61.0 \%$ \\
\hline sched & 130,199 & $37.3 \%$ & 33,911 & $37.9 \%$ & 254 & $0.20 \%$ & $0.75 \%$ & $\mathbf{5 9 . 5 \%}$ & $69.1 \%$ & $61.5 \%$ & 31,181 & $23.9 \%$ & $91.9 \%$ & $45.3 \%$ & $58.4 \%$ & $62.9 \%$ \\
\hline sched2 & 93,173 & $35.3 \%$ & 31,200 & $33.7 \%$ & 250 & $0.27 \%$ & $0.80 \%$ & $49.2 \%$ & $64.6 \%$ & $56.8 \%$ & 30,227 & $32.4 \%$ & $96.9 \%$ & $38.4 \%$ & $49.5 \%$ & $56.3 \%$ \\
\hline totinfo & 451,715 & $33.9 \%$ & 86,499 & $39.7 \%$ & 229 & $0.05 \%$ & $0.26 \%$ & $50.8 \%$ & $55.8 \%$ & $53.5 \%$ & 25,151 & $5.6 \%$ & $29.1 \%$ & $43.2 \%$ & $31.5 \%$ & $53.3 \%$ \\
\hline tcas & 40,883 & $37.3 \%$ & 11,377 & $33.3 \%$ & 112 & $0.27 \%$ & $0.98 \%$ & $46.2 \%$ & $70.5 \%$ & $47.5 \%$ & 5,846 & $14.3 \%$ & $51.4 \%$ & $39.4 \%$ & $53.1 \%$ & $47.7 \%$ \\
\hline Average: & & $35.4 \%$ & & $37.7 \%$ & & $0.37 \%$ & $1.73 \%$ & $48.0 \%$ & $60.7 \%$ & $52.5 \%$ & & $18.7 \%$ & $79.8 \%$ & $40.2 \%$ & $48.4 \%$ & $52.2 \%$ \\
\hline
\end{tabular}

TABLE III: $\left|O_{\mathrm{W}}\right|,\left|O_{\mathrm{T}}\right|$, and $\left|O_{\mathrm{M}}\right|$ denote the number of observations used by each of W-ORBS, T-ORBS, and MOAD, respectively. $\mu\left(W S_{k}\right), \mu\left(T S_{k}\right)$, and $\mu\left(M S_{k}\right)$ denote the mean slice size, given as a percentage of the original program's size, generated by each W-ORBS, T-ORBS and MOAD, respectively. Columns 9-11 and 15-17 show $\mu\left(M S_{k}\right)$ separately for each of the three models where the smallest mean for the three is shown in bold.

\begin{tabular}{|c|c|c|c|c|c|c|c|c|c|c|c|c|c|c|c|c|c|c|c|}
\hline \multirow{3}{*}{ Subject } & \multirow{3}{*}{$\begin{array}{l}\text { Deletion } \\
\text { Generation } \\
\text { Scheme }\end{array}$} & \multicolumn{6}{|c|}{$M S_{k}$ vs $W S_{k}$ (line) } & \multicolumn{6}{|c|}{$M S_{k}$ vs $T S_{k}$ (line) } & \multicolumn{6}{|c|}{$M S_{k}$ vs $T S_{k}(\mathrm{stmt})$} \\
\hline & & & & & & & & & D & & & & $\mathbb{B}$ & & 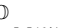 & & & & B \\
\hline & & $\mathrm{E}(\%)$ & $\mathrm{M}(\%)$ & $\mathrm{E}(\%)$ & $\mathrm{M}(\%)$ & $\mathrm{E}(\%)$ & $\mathrm{M}(\%)$ & $\mathrm{E}(\%)$ & $\mathrm{M}(\%)$ & $\mathrm{E}(\%)$ & $\mathrm{M}(\%)$ & $\mathrm{E}(\%)$ & $\mathrm{M}(\%)$ & $\mathrm{E}(\%)$ & $\mathrm{M}(\%)$ & $\mathrm{E}(\%)$ & $\mathrm{M}(\%)$ & $\mathrm{E}(\%)$ & $\mathrm{M}(\%)$ \\
\hline \multirow{2}{*}{ mbe } & hot & 3) & $11(18)$ & 2(3) & $13(2$ & 3) & 8) & $3(5)$ & $9(15)$ & $3(6)$ & 3) & $3(6)$ & $9(15)$ & 1(3) & $5(13)$ & 1(3) & $7(16)$ & 1(3) & $6(14)$ \\
\hline & 2-hot & 2(3) & $9(15)$ & 2(3) & $13(21)$ & 2(3) & $11(19)$ & 2(4) & $6(10)$ & $3(5)$ & $11(18)$ & $3(6)$ & $9(15)$ & 1(3) & $3(6)$ & 1(3) & $6(14)$ & 1(3) & $6(14)$ \\
\hline \multirow{2}{*}{ mug } & hot & 14) & 22) & $7(12)$ & $19(32$ & $8(14)$ & 140 & $0(1)$ & $6(11)$ & 2(4) & 15 & $0(0)$ & $7(1$ & $0(0)$ & $7(17)$ & $0(0)$ & $15(34)$ & $0(0)$ & $8(18)$ \\
\hline & 2-hot & $8(14)$ & $10(16)$ & $8(13)$ & $18(30)$ & $8(13)$ & $16(27)$ & $0(1)$ & $3(5)$ & $0(1)$ & $12(19)$ & $0(1)$ & $9(15)$ & $0(1)$ & 1(4) & $0(0)$ & $10(24)$ & $0(0)$ & $8(19)$ \\
\hline \multirow{2}{*}{ wc } & 1-hot & $5(11)$ & 18) & $4(9)$ & $16(36)$ & $4(10)$ & $13(28)$ & 1(2) & $6(13)$ & 2(4) & $15(34)$ & 1(3) & $11(24)$ & $0(1)$ & $4(14)$ & $0(1)$ & $11(34)$ & $0(1)$ & $6(21)$ \\
\hline & 2-hot & $5(12)$ & $6(13)$ & $5(12)$ & $13(28)$ & $4(10)$ & $10(23)$ & 1(3) & $3(8)$ & 2(6) & $11(25)$ & 1(3) & $9(20)$ & 1(4) & $2(7)$ & $1(4)$ & $8(25)$ & $0(2)$ & $5(17)$ \\
\hline \multirow{2}{*}{ prttok } & & $54(13)$ & $63(15)$ & $42(10)$ & $81(19)$ & $50(12)$ & $92(22)$ & 9(2) & $45(11)$ & 17 & $81(19)$ & $6(1)$ & $75(18)$ & $6(1)$ & $42(10)$ & $5(1)$ & $71(18)$ & $3(0)$ & $72(18)$ \\
\hline & 2-hot & $58(14)$ & $34(8)$ & $69(16)$ & $46(11)$ & $50(12)$ & $87(21)$ & $15(3)$ & $18(4)$ & $39(9)$ & $42(10)$ & $6(1)$ & $70(17)$ & $11(2)$ & $15(3)$ & $30(7)$ & $34(9)$ & $4(1)$ & $66(17)$ \\
\hline \multirow{2}{*}{ prttok2 } & 1-hot & (8) & 3 & 7(7) & 1280 & (7) & $61(15)$ & 12(3) & $8(7)$ & $14(3$ & 125 & 15 & 57 & $7(2)$ & $33(9)$ & 4( & 95 & $5(1)$ & $50(13)$ \\
\hline & 2-hot & $37(9)$ & $23(6)$ & $33(8)$ & $94(24)$ & $30(7)$ & $58(15)$ & 13(3) & $10(2)$ & $16(4)$ & $87(22)$ & $15(3)$ & $54(14)$ & $9(2)$ & $7(2)$ & $10(2)$ & $59(16)$ & $5(1)$ & $48(13)$ \\
\hline \multirow{2}{*}{ replace } & 1. & $3(22)$ & $110(21)$ & $1(20)$ & $171(33$ & $5(20)$ & 320 & $29(5)$ & $81(16)$ & $30(6$ & $156(30)$ & $40(8)$ & 122 & $15(3)$ & $63(13)$ & $13(2)$ & $111(23)$ & $13(2)$ & $91(19)$ \\
\hline & 2-hot & $124(24)$ & $66(13)$ & $112(22)$ & $94(18)$ & $106(20)$ & $133(26)$ & $34(6)$ & $32(6)$ & $48(9)$ & $86(16)$ & $38(7)$ & $121(23)$ & $25(5)$ & $17(3)$ & $28(6)$ & $52(11)$ & $13(2)$ & 91(19) \\
\hline \multirow{2}{*}{ sched } & & $33(11)$ & & $32(11)$ & $102(36)$ & & & 6( & 65 & 7( & & 1 & & $2-1$ & & 2( & & 2(1) & 28) \\
\hline & 2-hot & $35(12)$ & $37(13)$ & $30(10)$ & $72(25)$ & 33(11) & $85(30)$ & $7(2)$ & $26(9)$ & 9(3) & $69(24)$ & $7(2)$ & $77(27)$ & $3(1)$ & $27(10)$ & 2(1) & $60(23)$ & 2(1) & $76(30)$ \\
\hline \multirow{2}{*}{ sched2 } & 1-hot & $(15$ & $61(22)$ & (14) & 10 & $41(1$ & $81(29)$ & $5(1$ & $48(1$ & $7(2$ & 93 & 6( & 70 & $2(1$ & $45(1$ & 2 & 82 & 2(1) & $62(25)$ \\
\hline & 2-hot & $44(16)$ & $32(11)$ & $43(15)$ & $64(23)$ & $41(15)$ & $80(29)$ & $7(2)$ & 19(7) & $9(3)$ & $55(20)$ & $6(2)$ & $69(25)$ & 4(1) & $20(8)$ & $5(2)$ & $43(17)$ & 2(1) & $61(24)$ \\
\hline \multirow{2}{*}{ totinfo } & 1 & (11) & 7 & (10 & $5(2$ & ) & 78 & $3(1)$ & $42(13)$ & 7(2) & $65(2$ & $5(1)$ & 51( & $1(0)$ & $34(15)$ & $1(0)$ & 49 & $1(0)$ & $42(18)$ \\
\hline & 2-hot & $37(12)$ & $50(16)$ & $76(24)$ & $51(16)$ & $35(11)$ & $78(25)$ & 4(1) & 19(6) & $53(17)$ & $30(9)$ & $4(1)$ & $50(16)$ & 2(1) & $11(5)$ & $48(21)$ & $23(10)$ & $1(0)$ & $41(18)$ \\
\hline \multirow[b]{2}{*}{ tcas } & & $27(20)$ & & $21(15)$ & & & & 4( & & 3( & & & & & & & & & \\
\hline & 2-hot & $29(21)$ & $25(19)$ & $20(15)$ & $41(30)$ & $26(19)$ & $37(27)$ & $6(4)$ & $19(14)$ & 2(1) & $39(29)$ & 4(3) & $32(23)$ & 3(3) & $15(13)$ & $1(0)$ & $25(23)$ & $2(2)$ & $24(22)$ \\
\hline
\end{tabular}

TABLE IV: Average value of excess, denoted 'E', and miss, denoted 'M', in $M S_{k}$ when compared to $W S_{k}$ and $T S_{k}$. Columns 3-8 compare with $W S_{k}$ at the line level, Columns 9-14 compare with $T S_{k}$ at the line level, and finally Columns 15-20 compare with $T S_{k}$ at the statement (unit) level. The values in parentheses reflect the percentage of excess and miss compared to the number of lines or units in the original program.

duced by W-ORBS. The most significant difference occurs with the program wc when using the Logistic inference model trained with the 1-hot data. The size of the inferred slice is 2.3 times larger than that of the corresponding W-ORBS slice. On the other hand, there are inferred slices that are smaller than the corresponding W-ORBS slice. For example, the models trained by the configuration of (replace, Once Success, 2hot), (prttok, Logistic, 2-hot), and (totinfo, Logistic, 2-hot) inferred slices that have $96 \%, 99 \%$, and $93 \%$ of the W-ORBS slice size, respectively.

Next when compared with T-ORBS, the differences are smaller, which reflects T-ORBS producing slightly larger slices than W-ORBS on average. Over all programs MOAD produces slices that are $35.3 \%$ larger than T-ORBS. The most significant difference occurs for (tcas, Logistic, 1-hot) where the MOAD slice is 2.1 times larger. Because the T-ORBS slices are slightly larger than the W-ORBS slices there are again examples where the MOAD slice is smaller than the corresponding T-ORBS slice. We investigate the differences between MOAD slices and both T-ORBS and W-ORBS slices further with RQ3.

Turning to the question of how performance of the three models compares overall, Once Success $(\mathbb{O})$ generates smaller slices than the other two inference models (ANOVA, $p$ value $<0.0001$ ). Comparing the rows of Table III, Once Success produces the smaller slice in 17 of the 20 rows. Considering the overall impact of the training data, 2-hot produces the smaller average slice in 24 of the 30 cases.

Because of its dominance, we focus the remainder of the analysis on the 2-hot data. First, considering the Once Success model trained using the 2-hot observations, the size of the average inferred slice is $16 \%$ larger than the W-ORBS average and $7 \%$ larger than the T-ORBS average. This is due to how the inference algorithm Once Success works. No matter how rarely it happens, if the deletion of a unit does not affect the trajectory, Once Success learns to delete it when inferring a slice. This implies that the number of deletions monotonically increases as the set of observations is increased. Thus, Once Success trained with 2-hot data ubiquitously generates smaller slices than when trained with the 1-hot data.

Considering the Logistic model $(\mathbb{L})$, it also tends to generate smaller slices using the 2-hot data, doing so for nine of the ten subjects. This dominance illustrates that the model learns more dependency relations from the larger set of observations. Finally, the sizes of the slices generated by the Bayesian model 
$(\mathbb{B})$ show relatively minor variation as the size of the set of observations increases. That this is the most sophisticated of the models is thus evident. For example, the 1-hot data and 2-hot data each produce the smaller average for five of the subjects. Thus with more data, the estimated probability of preserving the slicing criterion may increase or decrease, depending on the observations. This results is echoed in the study of RQ4 in Section V-D.

Based on the data, we answer RQ2 as follows:

RQ2. Impact of the inference model: Among the three inference models, Once Success generates the smallest slices.

\section{Comparison with ORBS}

Table IV compares MOAD slices to those produced by the two implementations of ORBS. For each subject, deletion generation scheme, and inference model, we calculate excess and miss (defined in Section IV-E), to facilitate a more sophisticated comparison of each model's impact. The values shown in the table are averages for each program's slicing criteria; the values in parentheses are the corresponding percent of the number of lines or units in the original program. For both excess and miss, the smaller the number is, the more similar two slices are. Finally, note that it is only possible to compare with W-ORBS slices at the line level. In contrast, T-ORBS slices can be compared with MOAD slices at both the line level and the unit level.

Patterns evident in the data include that, when compared to W-ORBS, MOAD requires significantly fewer observations. For example, the number of 1-hot observations is orders of magnitude smaller than the number used by W-ORBS. Similarly, the 2-hot deletion generation scheme involves only $18.7 \%$ as many observations as used by W-ORBS. T-ORBS tends to use fewer observations than W-ORBS and thus, the values are closer. Overall, the number of of 1-hot observation is $1.7 \%$ of the number of T-ORBS observations, while the number of 2-hot observations is $79.8 \%$ of the number of observations used by T-ORBS.

The patterns for 1-hot and 2-hot extend into the individual models. As the number of observations increases from 1-hot to 2-hot, the value of miss for Once Success $(\mathbb{O})$ significantly decreases, while excess slightly increases. This tendency is repeated for the Logistic models $(\mathbb{L})$. However, miss barely changes for the Bayesian models $(\mathbb{B})$ which helps explain why the size of $\mathbb{B}$ slices shows little change in Table III.

Finally, turning to the three models, for all subjects and deletion generation schemes $\mathbb{O}$ yields the smallest values of miss. For example, it accurately deletes 11 to 30 more lines when compared to the other two models. While there are many cases where excess of $\mathbb{O}$ is larger than excess of other models, the difference is modest. Thus, among the inference models, $\mathbb{O}$ tends to generate slices more similar to those produced by W-ORBS and T-ORBS. Table V illustrates this by showing the average difference of miss and excess between (1) and the other two models. Values are computed over all

\begin{tabular}{r|rr|rr|rr}
\hline \multirow{2}{*}{ miss } & \multicolumn{2}{|c|}{$M S_{k}$ vs $W S_{k}$ (line) } & \multicolumn{2}{|c|}{$M S_{k}$ vs $T S_{k}$ (line) } & \multicolumn{2}{c}{$M S_{k}$ vs $T S_{k}$ (stmt) } \\
& $\mathbb{L}-\mathbb{O}$ & $\mathbb{B}-\mathbb{O}$ & $\mathbb{L}-\mathbb{O}$ & $\mathbb{B}-\mathbb{O}$ & $\mathbb{L}-\mathbb{O}$ & $\mathbb{B}-\mathbb{O}$ \\
\hline 1-hot & 29.8 & 11.2 & 36.5 & 14.6 & 24.9 & 11.0 \\
\hline 2-hot & 21.4 & 30.3 & 35.2 & 23.6 & 25.5 & 18.2 \\
\hline
\end{tabular}

\begin{tabular}{l|rr|rr|rr}
\hline \multirow{2}{*}{ excess } & \multicolumn{2}{|c|}{$M S_{k}$ vs $W S_{k}$ (line) } & \multicolumn{2}{|c|}{$M S_{k}$ vs $T S_{k}$ (line) } & \multicolumn{2}{c}{$M S_{k}$ vs $T S_{k}$ (stmt) } \\
& $\mathbb{L}-\mathbb{O}$ & $\mathbb{B}-\mathbb{O}$ & $\mathbb{L}-\mathbb{O}$ & $\mathbb{B}-\mathbb{O}$ & $\mathbb{L}-\mathbb{O}$ & $\mathbb{B}-\mathbb{O}$ \\
\hline 1-hot & -4.8 & -2.0 & 2.0 & 1.5 & -0.7 & -0.7 \\
\hline 2-hot & 1.9 & -4.4 & 6.4 & 1.7 & 2.8 & -1.3 \\
\hline
\end{tabular}

TABLE V: The difference in the average value of miss and excess between the three inference models. The upper table shows the data for miss while the lower table shows that for excess.

slicing criteria and subjects. It is clear from this data that for miss, $\mathbb{O}$ performs consistently and notably better than the other two models. Interestingly this dominance does not extend to excess where the values are smaller and more varied.

To gain confidence, we applied an ANOVA and then Tukey's HSD test [22] to the values of miss and excess ${ }^{1}$. The statistically significant results $(p<0.0001)$ show that miss for $\mathbb{O}$ is smaller than it is for $\mathbb{L}$ and $\mathbb{B}$. However, there is no significant differences between $\mathbb{L}$ and $\mathbb{B}$. The results for excess find $\mathbb{B}$ the smallest, followed by $\mathbb{O}$, and then $\mathbb{L}$.

Overall, the result shows that the Once Success model trained with the 2-hot observations generates slices that are not only compact but also the most similar to ORBS slices. However, there are some cases where the Logistic model produces the smallest slice, such as when slicing totinfo with the 2-hot data. However, such slices tend to have a high excess. Since an ORBS slice for a given slicing criteria is not necessary unique, we explicitly check the output trajectory of such small slices. Further investigation revealed that they are unable to preserve the trajectory of the targeting slicing criteria, which implies that the dependency inference was not sufficiently precise.

From the overall trends observed in size and similarity comparisons, we answer $\mathbf{R Q 3}$ as follows:

RQ3. Performance compared to ORBS: Using Once Success trained with the 2-hot data, MOAD requires less than one fifth of the observations compare to W-ORBS. At the same time the inferred slice is only $16 \%$ larger than the W-ORBS slice.

\section{Sampling effect}

RQ4 considers the tradeoff between the amount of training data used and the quality of the inference. To gain an initial impression for the data, Figure 1 shows two examples, taken from the programs replace and totinfo. In each of the six plots, the $x$-axis shows the size of the sample, while the $y$ axis shows the ratio of the average slice size, $\left|M S_{k}\right|$, to the original program size, $|P|$, where the values are averaged over all slicing criteria. Finally, the solid red (grey) and dashed blue horizontal lines represent the average slice size ratio produced by W-ORBS and T-ORBS, respectively. These six plots clearly

\footnotetext{
${ }^{1}$ The full details of Tukey's HSD results are available online at: https: //coinse.github.io/MOAD_Rdata_webpage/
} 


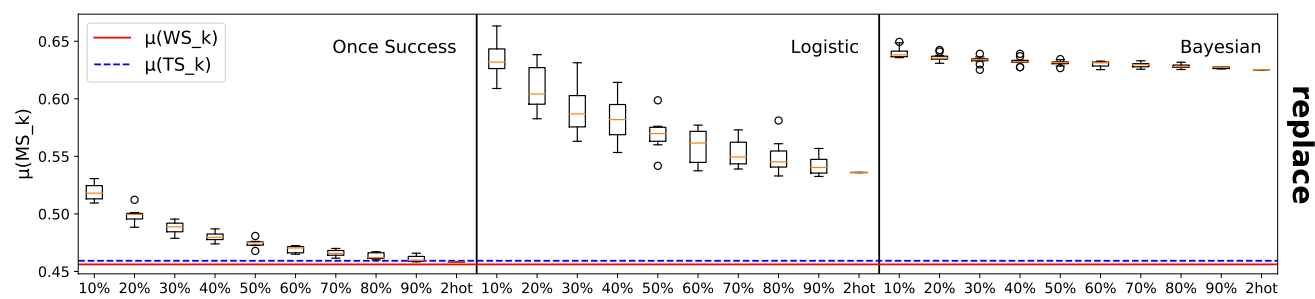

Sample size

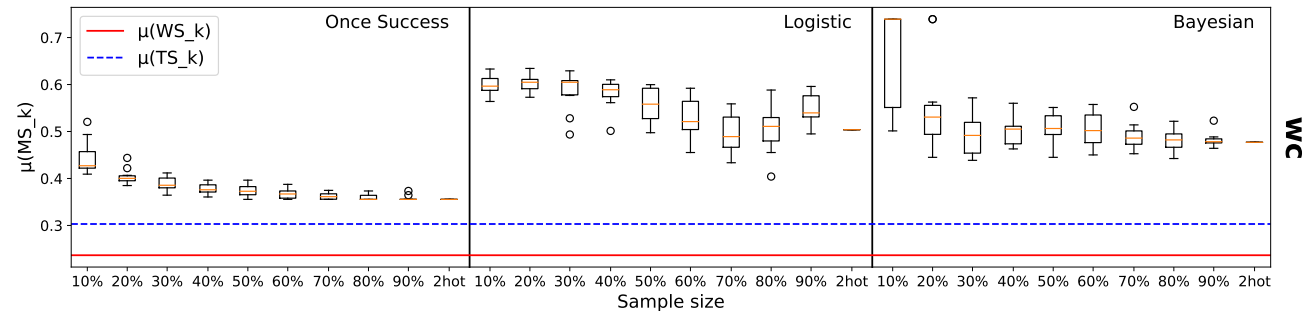

Fig. 1: The figure presents $\mu\left(M S_{k}\right)$ which represents the mean slice size given as a percentage of the original program's size, generated by MOAD using each size of the sample from 2-hot data. The boxplot shows the results of a trained model from 10 different random samplings. The red and blue line represents the ratio of the W-ORBS and T-ORBS slice size to the original program size, averaging by all slicing criteria of all subjects.

show that as the sample size increases the size of a MOAD slice approach that of the ORBS slices.

Because the plots indicate a fair amount of variation, we consider each model separately. To begin with, the left two plots for the Once Success model $\mathbb{O}$ suggest that it performs better when using $100 \%$ of the data. The reduction in size is substantial at small sample sizes, and it continues to decrease as the sample size increases. For example, the average of the first three slice size differences $(20 \%-10 \%, 30 \%-20 \%$, and $40 \%-30 \%$ ) is 4.4 times larger than the average of the last three slice size differences $(70 \%-80 \%, 80 \%-90 \%$, and $90 \%-100 \%)$. Note also that, when using only half of the 2-hot observations, (1) generates slices that are only $3.4 \%$ larger than when using all the data. Finally, the variance among different individual samples (the higher of the boxes) is relatively small, which suggests that $\mathbb{O}$ is robust against the stochastic sampling.

For the Logistic model, $\mathbb{L}$, the size of the slices also tends to decrease as the sample size increases, but the trend is not as strong as with $\mathbb{O}$. The $\mathbb{L}$ model also shows higher variance across samplings, when compared to other inference models. Similarly, while the Bayesian model, $\mathbb{B}$, tends to generate smaller slices with more observations, the median size fluctuates and the difference in slice size between samples is relatively small.

To gain additional statistical confidence, we applied ANOVA separately to each model ${ }^{2}$. In all three cases, the results are statistically significant $(p<0.0001)$. Applying Tukey's post-hoc test finds five equivalence classes of the mean slice sizes. The most useful findings are that using samples of $40 \%$ to $90 \%$ of the 2 -hot data produces mean slice sizes that are not statistically different. The same is true of the range $50 \%$ to $100 \%$, suggesting that using only half the data produces results essentially indistinguishable from using all of the data.

\footnotetext{
${ }^{2}$ The full details of ANOVA results are available online at: https://coinse github.io/MOAD_Rdata_webpage
}

For the $\mathbb{L}$ models, there is a similar band from $30 \%$ to $80 \%$, and two narrower bands from $60 \%$ to $90 \%$ and $80 \%$ to $100 \%$. These bands being narrower reflects the models being more sensitive to the amount of data that they are trained on.

Finally, the Bayesian model, $\mathbb{B}$, shows the greatest stability with all values from $20 \%$ to $100 \%$ being in the same band. Thus only when using a $10 \%$ sample does the model show inferior performance. This suggests that these models themselves are very robust against sampling variances. If it were possible to improve the size and the accuracy of slices produced by $\mathbb{B}$ models, the stability observed here may be a strong benefit of using $\mathbb{B}$ models.

We answer RQ4 as follows.

RQ4. Sampling effect: The high rate of reduction and high variability of the inferred slice size for small sample sizes indicates there is some sampling effect especially with Once Success. On the other hand, the wide bands show that it is possible to build high performing models using only a fraction of the training data. For example, Once Success infers slices $3.4 \%$ larger when using only half of the observations while the Bayesian model has similar performance using only $20 \%$ of the training data.

\section{Discussions AND FUTURE WORK}

\section{A. Once Success (O) vs. Critical Slicing vs. ORBS}

The Once Success model, $\mathbb{O}$ generates slices by deleting a line if the sub-program without the line preserves the trajectory. This is conceptually identical to critical slicing [23] and the slice using the pair $\langle 1$-hot, $\mathbb{O}\rangle$ is exactly the same as a critical slice. The results from Section V-C show that the slices from critical slicing and ORBS are similar to each other for the programs studied in this paper. The next step is to investigate whether this tendency is retained on larger programs with more complex dependence structures. Similarly, 
the ORBS slice using statement-level granularity might give a similar slice to the slice from $\mathbb{O}$.

\section{B. Advanced and adaptive deletion generation scheme}

The study reported here generates deletions to observe before observation starts. A more principled way would be to use Design of Experiments [24] to systematically select among the many possible deletions. This could also allow and utilise the sampling of more than two deletions per build and observe cycle, which could provide faster learning. Yet another approach would be to generate the deletion just-in-time (adaptively) with respect to the observations that have been made (e.g., via active learning [25]). Using the observation data, an interim model might be learned that can propose future deletions with the highest potential information gain.

\section{Alternative inference models}

Richer inference models could be used to model the relationships between units, and between criteria. Models such as Bayesian Networks [26] might be used to encode the conditional independencies between parts of programs and to infer the likelihood of program unit inclusion in slices based on the distributed joint distribution encoded therein. Markov Random Fields might also be tried to model the strength of association between program units as observed. Even if such probabilistic graphical models can be expected to perform well since they can capture the often graph-like dependency relations of software, more general statistical inference models (e.g. Gaussian processes [21]) should also be explored.

\section{Observation-based forward slicing}

There are two ways to slice the source code. A backward slice leaves the source code which influences the target slicing criteria. A forward slice leaves the source code that is influenced by the target slicing criteria. ORBS is a backward slicing method; it focuses on preserving the trajectory of the (single) slicing criterion. In contrast, MOAD uses a set of slicing criteria. Thus it can infer the forward slice when given sufficient observations. One possible approach is to compute the forward slice, $S_{c}$, of the slicing criteria, $c$, as the set of statements that contain the other criterion whose trajectory changed when $c$ has also been changed.

\section{E. Parallelization Opportunities}

Observational slicing brings many opportunities for parallelization. An excellent example is P-ORBS (Parallel ORBS) [27]. The implementation of P-ORBS is similar to that of W-ORBS except that it attempts to delete a set of windows sizes in parallel and then selects the largest deletion that produced the correct execution semantics. This enables the slicer to more quickly delete large blocks of code. While TORBS brings less inherent opportunity for parallelization, it is possible, for example, to consider the parallel deletion of a set of siblings in the tree. MOAD, by virtue of building training data from a set of independent executions has obvious parallelization opportunities. For example, looking at Algorithm 1, each iteration of the loop on Lines 4-9 is independent.

\section{F. Applications}

Future work will evaluate if the models we build can also be effectively used for tasks such as fault localization and fault comprehension [2], [28], [29]. It will also consider the tradeoffs between precision and the usefulness of these models (e.g., early comprehension for orientation may benefit from slightly less precise models that retain some situational context in the slice, whereas bug-location may benefit from more precise slices).

\section{RELATED WORK}

There have been multiple proposals to approximate dependencies by complementing a statically extracted graph with dynamic information [2], [28], [29]. A notable example is Baah et al. [2] who proposed to annotate traditional program dependence graphs (PDGs) with probabilities that capture dependence relations from dynamic execution of test cases. They extend the PDG with edges having conditional probabilities that relate states of child nodes to the states of their parents. Similarly, Feng and Gupta [28] (using Bayesian Networks) and Gong et al. [29] (using direct calculations of conditional probabilities) model the correctness of program statements (for fault localization) based on the control flow graph. Santelices and Harrold use probability models to predict the likely impact of changes in forward slicing [30] by augmenting static forward slices with relevance scores (labelling dependence edges in the interprocedural dependence graph with probabilities relating to coverage and propagation). While we also propose probabilistic modeling of dependencies of program elements we do not need an existing program dependence graph nor detailed information about program execution state. Our coarser modeling is thus more generally applicable whilst still useful, at least for slicing.

\section{CONCLUSION}

This paper introduces and studies a new technique for modeling program dependence based on dynamic observation. The long term goal of this work is to enable the reformulation of dynamic program dependency analysis into a probabilistic space using statistical inference models. Doing so should lower analysis costs. Furthermore, the cost is all upfront: once the model is built, inferring results is very inexpensive. At the same time, the reformulation aims to retain the strengths of existing purely dynamic dependency analysis (no need for prior static analysis, language agnostic, scalable, etc.).

To illustrate the value of this new technique the paper studies MOAD, which uses the inference model to produce (observation based) dynamic slices. The results, presented in Section V, illustrate the value of the probabilistic model. Specifically, MOAD can produce slices that are only $16 \%$ larger than the 1-minimal W-ORBS slices on average, from models built using only $18.7 \%$ of the observations required by W-ORBS. Furthermore, once the model is trained, the slicing cost is negligible. 


\section{REFERENCES}

[1] P. E. Livadas and P. K. Roy, "Program dependence analysis," in Proceedings of the International Conference on Software Maintenance 1992. Los Alamitos, California, USA: IEEE Computer Society Press, Nov. 1992, pp. 356-365.

[2] G. K. Baah, A. Podgurski, and M. J. Harrold, "The probabilistic program dependence graph and its application to fault diagnosis," IEEE Transactions on Software Engineering, vol. 36, no. 4, pp. 528-545, 2010.

[3] Zhifeng Yu and V. Rajlich, "Hidden dependencies in program comprehension and change propagation," in Proceedings 9th International Workshop on Program Comprehension. IWPC 2001, May 2001, pp. 293 299.

[4] D. Binkley, "Semantics guided regression test cost reduction," IEEE Transactions on Software Engineering, vol. 23, no. 8, pp. 498-516, 1997.

[5] K. B. Gallagher, "Using program slicing in software maintenance," Ph.D. dissertation, University of Maryland, Baltimore, Maryland, December 1989.

[6] Á. Hajnal and I. Forgács, "A demand-driven approach to slicing legacy cobol systems," Journal of Software: Evolution and Process, vol. 24, no. 1, pp. 67-82, 2012.

[7] R. Ettinger and M. Verbaere, "Untangling: A slice extraction refactoring," in Proceedings of the 3rd International Conference on Aspect-oriented Software Development, ser. AOSD '04. New York, NY, USA: ACM, 2004, pp. 93-101. [Online]. Available: http://doi.acm.org/10.1145/976270.976283

[8] R. Karim, F. Tip, A. Sochurkova, and K. Sen, "Platform-independent dynamic taint analysis for javascript," IEEE Transactions on Software Engineering, pp. 1-1, 2019.

[9] S. Jiang, C. McMillan, and R. Santelices, "Do programmers do change impact analysis in debugging?" Empirical Software Engineering, vol. 22, no. 2, pp. 631-669, Apr 2017. [Online]. Available: https://doi.org/10.1007/s10664-016-9441-9

[10] S. Horwitz, T. Reps, and D. W. Binkley, "Interprocedural slicing using dependence graphs," ACM Transactions on Programming Languages and Systems, vol. 12, no. 1, pp. 26-61, 1990.

[11] D. Binkley, N. Gold, M. Harman, S. Islam, J. Krinke, and S. Yoo, "ORBS: Language-independent program slicing," in Proceedings of the 22nd ACM SIGSOFT International Symposium on the Foundations of Software Engineering, ser. FSE 2014, 2014, pp. 109-120.

[12] _ " "ORBS and the limits of static slicing," in Proceedings of the 15th IEEE International Working Conference on Source Code Analysis and Manipulation, ser. SCAM 2015, September 2015, pp. 1-10.

[13] N. Gold, D. Binkley, M. Harman, S. Islam, J. Krinke, and S. Yoo, "Generalized observational slicing for tree-represented modelling languages," in Proceedings of the 11th Joint Meeting of the European Software Engineering Conference and the ACM SIGSOFT Symposium on the Foundations of Software Engineering, ser. ESEC/FSE 2017, 2017, pp. 547-558.

[14] D. Binkley, N. Gold, S. Islam, J. Krinke, and S. Yoo, "A comparison of tree- and line-oriented observational slicing," Empirical Software Engineering, Jan 2019. [Online]. Available: https://doi.org/10.1007/ s10664-018-9675-9

[15] S. Yoo, D. Binkley, and R. Eastman, "Observational slicing based on visual semantics," Journal of Systems and Software, vol. 129, pp. 60-78, July 2017.

[16] M. Weiser, "Program slices: Formal, psychological, and practical investigations of an automatic program abstraction method," Ph.D. dissertation, University of Michigan, Ann Arbor, MI, 1979.

[17] — , "Program slicing," IEEE Transactions on Software Engineering, vol. 10, no. 4, pp. 352-357, 1984.

[18] H. Do, S. G. Elbaum, and G. Rothermel, "Supporting controlled experimentation with testing techniques: An infrastructure and its potential impact." Empirical Software Engineering, vol. 10, no. 4, pp. 405-435, 2005.

[19] M. L. Collard, M. J. Decker, and J. I. Maletic, "srcML: An Infrastructure for the Exploration, Analysis, and Manipulation of Source Code: A Tool Demonstration," in Int'l Conf. Softw. Maintenance. IEEE, 2013, pp. 516-519.

[20] C. Lattner and V. Adve, "LLVM: A compilation framework for lifelong program analysis and transformation," San Jose, CA, USA, Mar 2004, pp. 75-88.

[21] C. E. Rasmussen, "Gaussian processes in machine learning," in Summer School on Machine Learning. Springer, 2003, pp. 63-71.
[22] J. W. Tukey, Exploratory Data Analysis: Limited Preliminary Ed. Addison-Wesley Publishing Company, 1970.

[23] R. A. DeMillo, H. Pan, and E. H. Spafford, "Critical slicing for software fault localization," in Proceedings of the 1996 ACM SIGSOFT International Symposium on Software Testing and Analysis, ser. ISSTA '96. New York, NY, USA: ACM, 1996, pp. 121-134.

[24] K. Chaloner and I. Verdinelli, "Bayesian experimental design: A review," Statistical Science, pp. 273-304, 1995.

[25] M. Zuluaga, G. Sergent, A. Krause, and M. Püschel, "Active learning for multi-objective optimization," in International Conference on Machine Learning, 2013, pp. 462-470.

[26] F. V. Jensen, Introduction to Bayesian Networks, 1st ed. Berlin, Heidelberg: Springer-Verlag, 1996.

[27] S. Islam and D. Binkley, "PORBS: A parallel observation-based slicer," in 24th International Conference on Program Comprehension (ICPC). IEEE, 2016, pp. 1-3.

[28] M. Feng and R. Gupta, "Learning universal probabilistic models for fault localization," in Proceedings of the 9th ACM SIGPLAN-SIGSOFT workshop on Program analysis for software tools and engineering. ACM, 2010, pp. 81-88.

[29] D. Gong, X. Su, T. Wang, P. Ma, and W. Yu, "State dependency probabilistic model for fault localization," Information and Software Technology, vol. 57, pp. 430-445, 2015.

[30] R. Santelices and M. J. Harrold, "Probabilistic slicing for predictive impact analysis," Georgia Institute of Technology, Tech. Rep. GITCERCS-10-10, 2010. 